important role in IFN production in B cells through regulation of TLR7/9 activation 2). We also identified that SLE patients with risk allele of SLC15A4 had tendency to show higher plasma $\mathrm{His}$ level, indicating His homeostasis could become a novel treatment target for SLE. Moreover, the inverse correlation of His level to SDI as well as OXPHOS signature suggests that His might play a key role for promoting organ damages in SLE.

References:

[1] Nat Genet. 2017;49:568. 2) Immunity. 2014;41:375. 3) Semin Arthritis Rheum. 2019;48:1142

Disclosure of Interests: : Yukiko Iwasaki: None declared, Yusuke Takeshima: None declared, Masahiro Nakano: None declared, Mineto Ota: None declared, Yasuo Nagafuchi: None declared, Akari Suzuki: None declared, Yuta Kochi: None declared, Tomohisa Okamura: None declared, Takaho Endo: None declared, Ichiro Miki: None declared, Kazuhiro Sakurada: None declared, Kazuhiko Yamamoto Grant/research support from: Astellas, BMS, MitsubishiTanabe, Pfizer, Ayumi, Takeda, Chugai, Eisai, Taisho Toyama, UCB, and ImmunoFuture, Keishi Fujio Grant/research support from: Astellas, BMS, MitsubishiTanabe, Pfizer, Ayumi, Takeda, Chugai, Eisai, Taisho Toyama, Eli Lilly, Sanofi, and UCB

DOI: 10.1136/annrheumdis-2020-eular.183

\section{THU0226 MESENCHYMAL STEM CELL TRANSPLANTATION AMELIORATES EXPERIMENTAL SJÖGREN'S SYNDROME BY DOWNREGUALTING MDSCS VIA COX2/PGE2 PATHWAY}

\section{G. Yao ${ }^{1}$, S. Wang ${ }^{1}$, L. Sun ${ }^{1} .{ }^{1}$ Nanjing University, Nanjing, China}

Background: Although mesenchymal stem cells (MSCs) transplantation have been demonstrated to be an effective therapeutic approach to treat experimental Sjögren's syndrome (ESS) ${ }^{1}$, the specific underlying mechanisms remain to be elucidated. Myeloid-derived suppressor cells (MDSCs) were significantly increased with decreased suppressive capacity during disease development in $\mathrm{ESS}^{2-3}$. However, the therapeutic effects and mechanisms by which MSCs regulating MDSCs in SS still remain unknown.

Objectives: Here we aim to explore whether regulation of MDSCs was responsible for the beneficial effects of MSC transplantation on SS.

Methods: The MSCs were infused intonon-obese diabetic (NOD) mice via the tail vein. The histological features of submandibular glands, lung, saliva flow rate were evaluated. The number and immune-suppressive activity of MDSCs, the subsets of MDSCs, polymorphonuclear MDSCs (PMN-MDSCs) and monocytic-MDSCs (M-MDSCs) in NOD mice were determined. The bone marrow cells under MDSCs differentiation conditions were co-cultured with or without MSCs. The COX2 inhibitor NS-398, anti-TGF- $\beta 1$, or anti-IFN- $\beta$ antibodies were added to coculture medium of MSCs and MDSCs induced from bone marrow cells respectively.

Results: We found that MDSCs in bone marrow and peripheral blood increased in ESS mice. MSC transplantation ameliorated SS-like syndrome and down-regulated the percentages of MDSCs, PMN-MDSCs and M-MDSCs and promoted their suppressive ability in ESS mice significantly (Figure 1). In vitro, MSCs could down-regulate the differentiation and up-regulate the suppressive ability of MDSCs. Mechanistically, MSCs inhibited the differentiation of MDSCs and PMN-MDSCs via secreting prostaglandin $E 2$, and inhibited the differentiation of M-MDSCs by secreting interferon- $\beta$ (Figure 2)
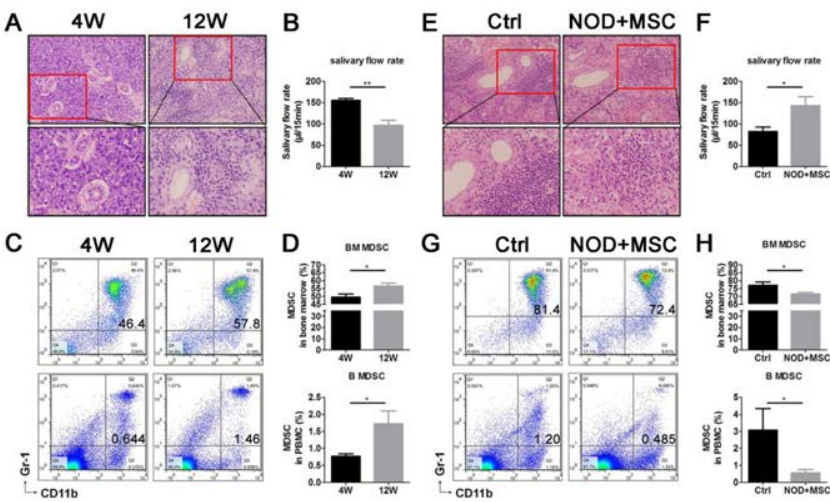

Figure 1. MSCs ameliorated SS symptoms and decreased MDSCs in NOD mice.

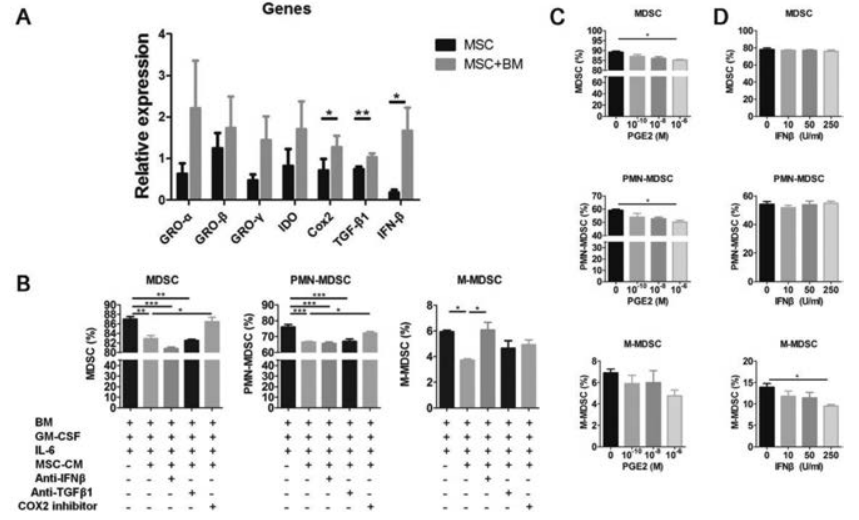

Figure 2. MSCs inhibited the differentiation of PMN-MDSCs and M-MDSCs by COX2/PGE2 and IFN- $\beta$ respectively.

Conclusion: Our findings suggested that MSCs alleviated SS-like symptoms by suppressing the aberrant accumulation and improving the suppressive function of MDSCs in ESS mice via COX2/PGE2 pathway.

References:

[1] Shi B, Qi J, Yao G, et al. Mesenchymal stem cell transplantation ameliorates Sjögren's syndrome via suppressing IL-12 production by dendritic cells. Stem Cell Res Ther, 2018; 9(1):308.

[2] Qi J, Li D, Shi G, et al. Myeloid-derived suppressor cells exacerbates Sjögren's syndrome by inhibiting Th2 immune responses. Mol Immunol, 2018; 101:251-258.

[3] Tian J, Rui K, Hong Y, et al. Increased GITRL impairs the function of myeloid-derived suppressor cells and exacerbates primary Sjögren's syndrome. J Immunol, 2019; 202(6):1693-1703.

Disclosure of Interests: None declared

DOI: 10.1136/annrheumdis-2020-eular.1391

\section{THU0227 CAFFEINE INTAKE MODULATES DISEASE ACTIVITY AND CYTOKINES LEVELS IN SYSTEMIC LUPUS ERYTHEMATOSUS PATIENTS}

V. Orefice ${ }^{1}$, F. Ceccarelli ${ }^{1}, 2$, C. Barbati ${ }^{1}$, R. Lucchetti ${ }^{1}$, G. Olivieri ${ }^{1}$, E. Cipriano ${ }^{1}$,

F. Natalucci ${ }^{1}$, C. Perricone ${ }^{2}$, F. R. Spinelli ${ }^{1}$, C. Alessandri ${ }^{1}$, G. Valesini ${ }^{1}$,

F. Conti'. ' 'Lupus Clinic, Rheumatology, Sapienza University of Rome, Rome,

Italy; ${ }^{2}$ University of Perugia, Department of Medicine, Rheumatology Unit,

Perugia, Italy

Background: Systemic lupus erythematosus (SLE) is an autoimmune disease mainly affecting women of childbearing age. The interplay between genetic and environmental factors may contribute to disease pathogenesis ${ }^{1}$. At today, no robust data are available about the possible contribute of diet in SLE. Caffeine, one of the most widely consumed products in the world, seems to interact with multiple components of the immune system by acting as a non-specific phosphodiesterase inhibitor ${ }^{2}$. In vitro dose-dependent treatment with caffeine seems to down-regulate mRNA levels of key inflammation-related genes and similarly reduce levels of different pro-inflammatory cytokines $^{3}$.

Objectives: We evaluated the impact of caffeine consumption on SLE-related disease phenotype and activity, in terms of clinimetric assessment and cytokines levels.

Methods: We performed a cross-sectional study, enrolling consecutive patients and reporting their clinical and laboratory data. Disease activity was assessed by SLE Disease Activity Index 2000 (SLEDAI-2k) ${ }^{4}$. Caffeine intake was evaluated by a 7-day food frequency questionnaire, including all the main sources of caffeine. As previously reported, patients were divided in four groups according to the daily caffeine intake: $<29.1 \mathrm{mg} /$ day (group 1), $29.2-153.7 \mathrm{mg} /$ day (group 2), $153.8-376.5 \mathrm{mg} /$ day (group 3) and $>376.6 \mathrm{mg} /$ day (group 4$)^{5}$. At the end of questionnaire filling, blood samples were collected from each patient to assess cytokines levels. These were assessed by using a panel by Bio-Plex assays to measure the levels of IL-6, IL-10, IL-17, IL-27, IFN- $\gamma$, IFN- $\alpha$ and Blys.

Results: We enrolled 89 SLE patients (F/M 87/2, median age 46 years, IQR 14; median disease duration 144 months, IQR 150). The median intake of caffeine was $195 \mathrm{mg} /$ day (IQR 160.5). At the time of the enrollment, 8 patients $(8.9 \%)$ referred a caffeine intake $<29.1 \mathrm{mg} /$ day (group 1$), 27$ patients $(30.3 \%)$ between 29.2 and $153.7 \mathrm{mg} /$ day (group 2), 45 patients (51\%) between 153.8 